\title{
Insights into the molecular mechanisms of bradycardia-triggered arrhythmias in long QT-3 syndrome
}

\author{
Colleen E. Clancy, Michihiro Tateyama, and Robert S. Kass \\ Department of Pharmacology, College of Physicians and Surgeons, Columbia University, New York, New York, USA
}

\begin{abstract}
Congenital long QT syndrome is a rare disease in which the electrocardiogram QT interval is prolonged due to dysfunctional ventricular repolarization. Variant 3 (LQT-3) is associated with mutations in $S C N 5 A$, the gene coding for the heart $\mathrm{Na}^{+}$channel $\alpha$ subunit. Arrhythmias in LQT-3 mutation carriers are more likely to occur at rest, when heart rate is slow. Several LQT-3 $\mathrm{Na}^{+}$channel mutations exert their deleterious effects by promoting a mode of $\mathrm{Na}^{+}$channel gating wherein a fraction of channels fails to inactivate. This gating mode, termed "bursting," results in sustained macroscopic inward $\mathrm{Na}^{+}$channel current $\left(I_{\text {sus }}\right)$, which can delay repolarization and prolong the QT interval. However, the mechanism of heart-rate dependence of $I_{\text {sus }}$ has been unresolved at the single-channel level. We investigate an LQT-3 mutant (Y1795C) using experimental and theoretical frameworks to elucidate the molecular mechanism of $I_{\text {sus }}$ rate dependence. Our results indicate that mutation-induced changes in the length of time mutant channels spend bursting, rather than how readily they burst, determines $I_{\text {sus }}$ inverse heart-rate dependence. These results link mutation-induced changes in $\mathrm{Na}^{+}$channel gating mode transitions to heart rate-dependent changes in cellular electrical activity underlying a key LQT-3 clinical phenotype.

This article was published online in advance of the print edition. The date of publication is available

from the JCI website, http://www.jci.org. J. Clin. Invest. 110:1251-1262 (2002). doi:10.1172/JCI200215928.
\end{abstract}

\section{Introduction}

Patient genotyping is becoming increasingly common as a method to search for molecular explanations of seemingly idiopathic syndromes $(1,2)$. However, it is difficult to make the connection between gene defects and abnormal function at the cellular level that underlies distinct syndromes. Cystic fibrosis, idiopathic ventricular fibrillation, and long QT syndrome are examples of clinical syndromes that arise from defects in ion channels (3-7). Ion channel mutations may exert their deleterious effects through alterations in gating kinetics, loss of ion selectivity, or dominant negative current suppression $(2,8,9)$. Investigation of inherited defects in ion channels may lead to improved understanding of the mechanistic basis of disease.

Congenital long QT syndrome, a rare disease in which the QT interval of the electrocardiogram is prolonged due to dysfunctional ventricular repolarization,

Received for publication May 14, 2002, and accepted in revised form August 14, 2002.

Address correspondence to: Robert S. Kass, Department of Pharmacology, Columbia University College of Physicians and Surgeons, 630 West 168th Street, New York, New York 10032, USA. Phone: (212) 305-7444; Fax: (212) 342-2703;

E-mail: rsk20@columbia.edu.

Colleen E. Clancy and Michihiro Tateyama contributed equally to this work.

Conflict of interest: No conflict of interest has been declared. Nonstandard abbreviations used: long QT syndrome variant 3 (LQT-3); $\mathrm{Na}^{+}$current $\left(I_{\mathrm{Na}}\right)$; wild type (WT); sustained $\mathrm{Na}^{+}$channel inward current $\left(I_{\text {sus }}\right)$; action potential (AP); AP duration (APD); human embryonic kidney (HEK); tetrodotoxin (TTX); mean open time (MOT). is associated with syncope and sudden death $(10,11)$. Congenital long QT syndrome is associated with mutations in genes coding for at least six ion channel subunits (11). These mutations result in cardiac events with various triggers (10). Long QT syndrome variant 3 (LQT-3) is associated with mutations in SCN5A, the gene coding for the $\alpha$ subunit of the voltage-gated $\mathrm{Na}^{+}$ channel (7), and LQT-3 mutation carriers are at greater risk of cardiac events during rest or bradycardia than during exercise when heart rate is elevated (10). Rhythm disturbances arising from some LQT-3-linked SCN5A defects stem from a gain of $\mathrm{Na}^{+}$channel function that promotes the conduction of $\mathrm{Na}^{+}$ions for prolonged periods at depolarized membrane potentials (7, $12,13)$. Most defects have been shown to reduce the stability of inactivation of the cardiac $\mathrm{Na}^{+}$current $\left(I_{\mathrm{Na}}\right)$ during prolonged depolarization, when wild-type (WT) channels typically enter an absorbing inactivation state $(7,12,13)$. This mutation-induced destabilization of inactivation increases sustained $\mathrm{Na}^{+}$channel inward current $\left(I_{\text {sus }}\right)$ during the action potential (AP) plateau, which disrupts the delicate balance of membrane currents required for regulation of AP duration (APD). Excess inward current via inactivation-deficient $\mathrm{Na}^{+}$ channels can thus prolong APD and facilitate the development of arrhythmogenic early afterdepolarizations $(14,15)$ at the cellular level. These cellular effects are consistent with mutation-induced QT prolongation and arrhythmia risk at the systems level.

Patients afflicted with SCN5A-linked LQT-3 have been observed to experience cardiac events that are strongly dependent on heart rate $(1,16)$. At slow heart 
rates during rest or relaxation, patient phenotypes are marked by prolonged QT intervals and susceptibility to polymorphic tachyarrhythmias (16). To date, the molecular mechanism by which the severity of LQT-3 phenotype worsens at slow heart rates is not known at the level of mutation-induced changes in single ion channel kinetic properties. Previous work $(14,15)$ has demonstrated mutation-induced rate dependence of APD using a theoretical modeling approach, but has lacked the required precision for detailed analyses of the molecular mechanism of $I_{\text {sus }}$ stimulation-rate dependence. Previously, the paucity of single-channel records precluded extraction of actual time constants of entry into and exit from the burst mode. As a result, a thorough understanding of the rate dependence of channel bursting has been elusive.

Here we combine theoretical and experimental approaches to illuminate the mechanism of the rate dependence of $I_{\text {sus }}$ at the cellular level through analyses of experimentally measured mutation-altered singlechannel gating. We first focused on an LQT-3 mutation (Y1795C) that had been previously described at the whole-cell level (17). We selected the Y1795C mutation because QT prolongation is pronounced at slow heart rates in mutation carriers, and previous analysis revealed a strong inverse pulse-rate dependence of Y1795C channel $I_{\text {sus }}$ amplitude (17). We performed detailed kinetic analyses of single Y1795C channels and used these data to extract transition rate constants of bursting of single $\mathrm{Na}^{+}$channels. These rate constants were then incorporated into a Markov model of $I_{\mathrm{Na}}(15)$ that recapitulates the features of experimentally measured current amplitude and rate dependence.

Our computational analysis suggests that the amount of time mutant channels spend bursting (burst mode dwell time), rather than how readily they burst (latency to burst), is primarily responsible for rate-dependent changes in single-channel bursting and macroscopic $I_{\text {sus. }}$. This prediction was tested and validated by the analysis of another LQT-3 mutation, $\triangle \mathrm{KPQ}$. These results provide an explanation of the molecular mechanism for bradycardia-induced QT prolongation in patients carrying LQT-3 mutations.

\section{Methods}

\section{Experimental methods}

Expression of recombinant $\mathrm{Na}^{+}$channels. $\mathrm{Na}^{+}$channels were expressed in human embryonic kidney (HEK) 293 cells. Transient transfection was carried out with equal amounts of WT or mutant $\mathrm{Na}^{+}$channel $\alpha$ subunit cDNA with human $\beta_{1}$ cDNA subcloned into the pcDNA3.1(+) vector (Invitrogen Corp., San Diego, California, USA) along with CD8, a commercially available reporter gene (EBO-pCD vector; American Type Culture Collection, Rockville, Maryland, USA). Total cDNA was $2.5 \mu \mathrm{g}$. We used a previously described lipofection procedure (18) to transfect cells. CD8-positive cells, identified using Dynabeads
(M-450; Dynal Biotech, Oslo, Norway), were patch clamped 48 hours after transfection.

\section{Electrophysiology}

Whole-cell recordings. Membrane currents were measured using whole-cell and single-channel patch clamp procedures, with Axopatch 200B amplifiers (Axon Instruments Inc., Foster City, California, USA). Capacity current and series resistance compensation were carried out using analogue techniques according to the amplifier manufacture (Axon Instruments Inc.). All measurements were obtained at room temperature $\left(22^{\circ} \mathrm{C}\right)$. Macroscopic whole-cell $\mathrm{Na}^{+}$current was recorded using the following solutions, shown in $\mathrm{mmol} / \mathrm{l}$. The internal solution contained 50 aspartic acid, $60 \mathrm{CsCl}, 5 \mathrm{Na}_{2} \mathrm{ATP}$, 11 EGTA, $10 \mathrm{HEPES}, 1 \mathrm{CaCl}_{2}$, and $1 \mathrm{MgCl}_{2}$, at $\mathrm{pH} 7.4$ adjusted with $\mathrm{CsOH}$. The external solution contained $130 \mathrm{NaCl}, 2 \mathrm{CaCl}_{2}, 5 \mathrm{CsCl}, 1.2 \mathrm{MgCl}_{2}, 10$ HEPES, and 5 glucose, at $\mathrm{pH} 7.4$ adjusted with $\mathrm{CsOH}$. The voltage dependence of inactivation was determined after application of conditioning pulses (500 milliseconds) applied once every 2 seconds to a series of voltages. In experiments designed to measure the voltage dependence of activation, external $\mathrm{Na}^{+}$was reduce to $30 \mathrm{mM}$ using $\mathrm{N}$-methyl glucamine as a $\mathrm{Na}^{+}$substitute. In experiments designed to test for sustained currents, tetrodotoxin (TTX) was applied at high concentrations (30 $\mu \mathrm{M})$ to block expressed $\mathrm{Na}^{+}$channel currents and reveal background currents, which were then subtracted digitally. Holding potential was $-100 \mathrm{mV}$ unless otherwise noted in figure captions.

Single-channel experiments. Single-channel experiments were carried out using the cell-attached configuration (seal resistance greater than $10 \mathrm{G} \Omega$ ). Test pulses $(-30$ $\mathrm{mV}, 100$ milliseconds) were applied every 0.5 seconds from a holding potential of $-120 \mathrm{mV}$. Single-channel currents were low-pass filtered (using a $5 \mathrm{kHz}$ cutoff frequency) and digitized at a sampling frequency of 20 $\mathrm{kHz}$. Capacitative and leak currents were eliminated by digital subtraction of averaged null sweeps. Idealization of single-channel currents and measurement of open time were carried out with the SKM program (QuB software suite; Research Foundation of State University of New York, Buffalo, New York, USA) $(19,20)$. Open probability $\left(P_{\mathrm{o}}\right)$ for one or two channel patches was calculated by the equation:

\section{Equation 1}

$$
P_{0}=1-(b / s)^{1 / n}
$$

The variables $b, s$, and $n$ represent the number of blank sweeps, total sweeps, and channels, respectively (21). We used the same formulation to determine the frequency of bursting. Burst frequency was estimated as above by substituting $b$ (in this case, the number of sweeps without bursting) and $s$, the total number of sweeps studied. Here the number of channels per patch $(n)$ was estimated by counting overlapping unitary current from $1,000-$ 2,000 total sweeps; the count was always less than 11 . 
$\mathrm{Na}^{+}$channel model

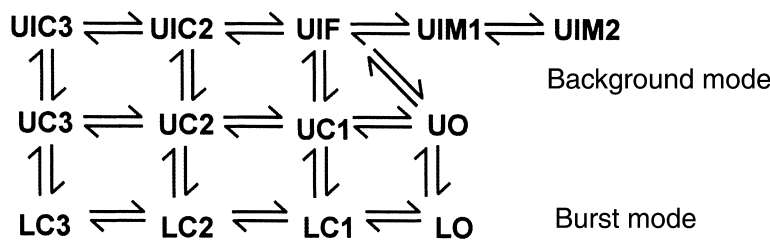

Figure 1

Markov model of the cardiac $\mathrm{Na}^{+}$channel. The channel model contains background (upper nine states) and burst (lower four states) gating modes. The burst mode reflects a population of channels that transiently fail to inactivate.

All analysis was carried out using Excel (Microsoft Corp., Redmond, Washington, USA) and Origin 6.1 (Microcal Software Inc., Northampton, Massachusetts, USA). Data are represented as mean \pm SEM. Statistical significance was determined using an unpaired Student $t$ test; $P<0.05$ was considered statistically significant.

Computational methods. The Markov formulation of $I_{\mathrm{Na}}$ (Figure 1) is an extension of the Clancy-Rudy (14) model previously developed with recent modifications (15).

Model framework: background and bursting modes. Mutant channels differ from WT channels in two distinct ways, as evidenced by experimental recordings. Mutant channels have altered mean open times (MOTs) compared with WT channels, due to altered rates of channel open-state inactivation (UO $\rightarrow$ UIF; see definitions below). Mutant channels in this study also burst more often than did WT channels.

The Markov model of $I_{\mathrm{Na}}$ is shown in Figure 1. The model contains two possible modes of gating, a background (dispersed) mode and a burst mode. The background mode includes the upper nine states, consisting of three closed states (UC3, UC2, UC1), a conducting open state (UO), a fast inactivation state (UIF), and two intermediate inactivation states (UIM1 and UIM2) that are required to reproduce the complex fast and slow recovery features of inactivation. The UIM1 state acts as a channel "sink" in which the majority of channels reside but are unable to recover and reopen during depolarization. Channels enter the UIM2 state via slow transitions. Channel closed-state inactivation is achieved via the inclusion of two closed-inactivation states (UIC2 and UIC3). The UO $\rightarrow$ UIF transition is adjusted accordingly in modeling mutant channels in order to reflect the changes in MOT observed experimentally. These changes are also reflected in the time course of the onset of macroscopic current inactivation (current decay) during depolarization. The lower four states in Figure 1 (prefixed with "L," denoting "lower") correspond to a burst mode of gating of channels that lack inactivation. Single-channel experiments suggest that transitions between these modes are voltage independent since bursting may persist through a series of pulses, depolarizing to $-30 \mathrm{mV}$ (over a period of 100 milliseconds) and repolarizing to $-120 \mathrm{mV}$ (over a period of 400 milliseconds) (see Figure 5). Hence transition rates between upper and lower states represent a probability of transition between the two modes of gating.

Modeling macroscopic currents. Macroscopic current density is given by:

\section{Equation 2}

$$
I_{\mathrm{s}}=G_{\mathrm{s}} \times P_{\mathrm{o}, \mathrm{s}} \times\left(V_{\mathrm{m}}-E_{\mathrm{rev}}\right) \text { where } G_{\mathrm{s}}=\sigma \times g_{\mathrm{s}}
$$

The variables $P_{\mathrm{o}, \mathrm{s}}$ are the sum of all channel open probabilities, $V_{\mathrm{m}}$ is the membrane potential, and $E_{\mathrm{rev}}$ is the reversal potential. $G_{s}$ is the maximum membrane conductance: channel density $(\sigma)$ times the unitary channel conductance $\left(g_{\mathrm{s}}\right)$.

The changes in channel state probabilities are described by first order differential equations. Assuming $N$ discrete channel states, the probability of the channel residing in a particular state $P_{i}$ at any time satisfies:

\section{Equation 3}

$$
\begin{gathered}
d P_{i} / d t=\sum_{j=1}^{N}\left[k_{j i} \times P_{j}\left(t, V_{m}\right)\right]-\sum_{j=1}^{N}\left[k_{i j} \times P_{i}\left(t, V_{m}\right)\right] \\
\text { for } i=1,2, \ldots, N-1 ; i \neq j \text { and } \sum_{j=1}^{N} P_{i}=1
\end{gathered}
$$

The voltage-dependent $\left(V_{\mathrm{m}}\right.$-dependent) rate constants $k_{i j}$ describe the transition from state $i$ to state $j$. Initial conditions are obtained by finding values for state probabilities from the steady-state equation:

\section{Equation 4}

$$
d P_{i} / d t=0
$$

Assuming that the probability in each state $i$ at time $t_{\mathrm{n}}$ is:

\section{Equation 5}

$$
\begin{aligned}
& P_{1}\left(t_{\mathrm{n}}\right), P_{2}\left(t_{\mathrm{n}}\right), \ldots, P_{\mathrm{N}}\left(t_{\mathrm{n}}\right) \\
& \text { then at } t_{\mathrm{n}+1}=t_{\mathrm{n}}+\Delta t \\
& P_{i}\left(t_{\mathrm{n}+1}\right)=P_{i}\left(t_{\mathrm{n}}\right)+\Delta P_{i}
\end{aligned}
$$

$\Delta P_{i}$ is calculated at each time step. Picard iterates are computed by a second-order Runge-Kutta procedure to obtain the dynamic values of $P_{\mathrm{i}}$. The steady-state probability is used for $P_{\mathrm{i}}\left(t_{0}\right)$ by allowing simulated cell to rest without stimulus current until equilibrium is achieved (no change in computed parameters).

All the simulations were encoded in $\mathrm{C} / \mathrm{C}^{++}$. Simulations were implemented (double precision) on an Apple Macintosh PowerBook with a $500-\mathrm{MHz}$ Motorola G4 processor running OS X (Apple Computers, Cupertino, California, USA). A time step of 0.005 milliseconds was used.

Extraction of burst mode rate constants from experimental recordings. We used experimentally recorded macroscopic current and single-channel records for model parameter optimization. The single-channel records allowed for derivation of theoretical rate constants of 


\section{Table 1}

$\mathrm{Na}^{+}$channel model parameters

$I_{\mathrm{Na}}$

$I_{\mathrm{Na}}=G_{\mathrm{Na}} \times P_{\mathrm{o}} \times\left(V_{\mathrm{m}}-E_{\mathrm{Na}}\right)$

$P_{\mathrm{o}}=$ sum of open probabilities of $I_{\mathrm{Na}}$

$G_{\mathrm{Na}}=8.5$ millisiemens $/ \mu \mathrm{F}$

$E_{\mathrm{Na}}=(\mathrm{R} \times \mathrm{T} /$ /Faraday's constant $) \times \ln \left([\mathrm{Na}]_{\text {out }} /[\mathrm{Na}]_{\text {in }}\right)$

Transition rates: WT channel $\left(\mathrm{ms}^{-1}\right)$

$\mathrm{xC} 3 \rightarrow \mathrm{xC2}$

$x \mathrm{C} 2 \rightarrow \mathrm{xC} 1$

$\mathrm{xC} 1 \rightarrow \mathrm{xO}$

$\mathrm{UIC} 3 \rightarrow \mathrm{UIC2}$

$\mathrm{UIC2} \rightarrow \mathrm{UIF}$

$\mathrm{xC2} \rightarrow \mathrm{xC} 3$

$\mathrm{xC} 1 \rightarrow \mathrm{xC2}$

$\mathrm{xO} \rightarrow \mathrm{xC} 1$

$\mathrm{UIC2} \rightarrow \mathrm{UIC3}$

$\mathrm{UIF} \rightarrow \mathrm{UIC2}$

$\mathrm{UO} \rightarrow \mathrm{UIF}$

$\mathrm{UIF} \rightarrow \mathrm{UO}$

$\mathrm{UIF} \rightarrow \mathrm{UC} 1$

$\mathrm{UIC2} \rightarrow \mathrm{UC} 2$

$\mathrm{UIC} 3 \rightarrow \mathrm{UC} 3$

$\mathrm{UC} 1 \rightarrow \mathrm{UIF}$

$\mathrm{UC2} \rightarrow \mathrm{UIC2}$

$\mathrm{UC} 3 \rightarrow \mathrm{UIC} 3$

$\mathrm{UIF} \rightarrow$ UIM1

$\mathrm{UIM} 1 \rightarrow \mathrm{UIF}$

$\mathrm{UIM} 1 \rightarrow \mathrm{UIM} 2$

$\mathrm{UIM} 2 \rightarrow \mathrm{UIM} 1$

$\alpha 11=3.802 /\left[0.1027 \times \exp \left(-V_{m} / 17.0\right)+0.20 \times \exp \left(-V_{m} / 150\right)\right]$

$\alpha 12=3.802 /\left[0.1027 \times \exp \left(-V_{m} / 15.0\right)+0.23 \times \exp \left(-V_{m} / 150\right)\right]$

$\alpha 13=3.802 /\left[0.1027 \times \exp \left(-V_{m} / 12.0\right)+0.25 \times \exp \left(-V_{m} / 150\right)\right]$

$\alpha 11=3.802 /\left[0.1027 \times \exp \left(-V_{m} / 17.0\right)+0.20 \times \exp \left(-V_{m} / 150\right)\right]$

$\alpha 12=3.802 /\left[0.1027 \times \exp \left(-V_{m} / 15.0\right)+0.23 \times \exp \left(-V_{m} / 150\right)\right]$

$\beta 11=0.77 \times \exp \left(-\mathrm{V}_{\mathrm{m}} / 20.3\right)$

$\beta 12=0.77 \times \exp \left(-\left(V_{m}-5\right) / 20.3\right)$

$\beta 13=\left(0.17 \times \exp \left[-\left(\bigvee_{m}-10\right) / 20.3\right)\right]$

$\beta 11=0.77 \times \exp \left(-V_{m} / 20.3\right)$

$\beta 12=0.77 \times \exp \left[-\left(V_{m}-5\right) / 20.3\right]$

$\alpha 2=2.04 \times \exp \left(V_{m} / 29.68\right)$

$\beta 2=(\alpha 13 \times \alpha 2 \times \alpha 3) /(\beta 13 \times \beta 3)$

$\alpha 3=3.7933 \times 10^{-7} \times \exp \left(-V_{\mathrm{m}} / 7.7\right)$

$\alpha 3=3.7933 \times 10^{-7} \times \exp \left(-V_{\mathrm{m}} / 7.7\right)$

$\alpha 3=3.7933 \times 10^{-7} \times \exp \left(-V_{\mathrm{m}} / 7.7\right)$

$\beta 3=\left(0.0084+0.00002 \times V_{m}\right)$

$\beta 3=\left(0.0084+0.00002 \times V_{m}\right)$

$\beta 3=\left(0.0084+0.00002 \times V_{m}\right)$

$\alpha 4=\alpha 2 / 100$

$\beta 4=\alpha 3$

$\alpha 5=\alpha 2 /\left(9.5 \times 10^{4}\right)$

$\beta 5=\alpha 3 / 50$

Transition rates between modes are: background to burst $(U \rightarrow L)=1 \times 10^{-7} \mathrm{~ms}^{-1}$; burst to background $(\mathrm{L} \rightarrow \mathrm{U})=3.8 \times 10^{-3} \mathrm{~ms}^{-1}$.

Transition rates: $\mathrm{Y} 1795 \mathrm{C}$ channel $\left(\mathrm{ms}^{-1}\right)$

$\mathrm{xC} 3 \rightarrow \mathrm{xC2}$

$\mathrm{xC2} \rightarrow \mathrm{xC} 1$

$\mathrm{xC} 1 \rightarrow \mathrm{xO}$

$\mathrm{UIC} 3 \rightarrow \mathrm{UIC2}$

$\mathrm{UIC2} \rightarrow \mathrm{UIF}$

$\mathrm{xC2} \rightarrow \mathrm{xC} 3$

$\mathrm{xC} 1 \rightarrow \mathrm{xC2}$

$\mathrm{xO} \rightarrow \mathrm{xC} 1$

$\mathrm{UIC2} \rightarrow \mathrm{UIC3}$

$\mathrm{UIF} \rightarrow \mathrm{UIC2}$

$\mathrm{UO} \rightarrow \mathrm{UIF}$

$\mathrm{UIF} \rightarrow \mathrm{UO}$

$\mathrm{UIF} \rightarrow \mathrm{UC} 1$

$\mathrm{UIC2} \rightarrow \mathrm{UC2}$

$\mathrm{UIC} 3 \rightarrow \mathrm{UC} 3$

$\mathrm{UC} 1 \rightarrow \mathrm{UIF}$

$\mathrm{UC2} \rightarrow \mathrm{UIC2}$

$\mathrm{UC} 3 \rightarrow \mathrm{UIC} 3$

UIF $\rightarrow$ UIM1

UIM $1 \rightarrow$ UIF

$\mathrm{UIM} 1 \rightarrow \mathrm{UIM} 2$

$\mathrm{UIM} 2 \rightarrow \mathrm{UIM} 1$

$\alpha 11=3.802 /\left[0.1027 \times \exp \left(-V_{m} / 17.0\right)+0.20 \times \exp \left(-V_{m} / 150\right)\right]$

$\alpha 12=3.802 /\left[0.1027 \times \exp \left(-V_{m} / 15.0\right)+0.23 \times \exp \left(-V_{m} / 150\right)\right]$

$\alpha 13=3.802 /\left[0.1027 \times \exp \left(-V_{m} / 12.0\right)+0.25 \times \exp \left(-V_{m} / 150\right)\right]$

$\alpha 11=3.802 /\left[0.1027 \times \exp \left(-V_{m} / 17.0\right)+0.20 \times \exp \left(-V_{m} / 150\right)\right]$

$\alpha 12=3.802 /\left[0.1027 \times \exp \left(-V_{m} / 15.0\right)+0.23 \times \exp \left(-V_{m} / 150\right)\right]$

$\beta 11=0.77 \times \exp \left(-V_{m} / 20.3\right)$

$\beta 12=0.77 \times \exp \left[-\left(V_{m}-5\right) / 20.3\right]$

$\beta 13=\left(0.535 \times \exp \left[-\left(\mathrm{V}_{\mathrm{m}}-10\right) / 20.3\right)\right]$

$\beta 11=0.77 \times \exp \left(-V_{\mathrm{m}} / 20.3\right)$

$\beta 12=0.77 \times \exp \left[-\left(V_{m}-5\right) / 20.3\right]$

$\alpha 2=2.04 \times \exp \left(\mathrm{V}_{\mathrm{m}} / 29.68\right) / 2$

$\beta 2=(\alpha 13 \times \alpha 2 \times \alpha 3) /(\beta 13 \times \beta 3)$

$\alpha 3=3.7933 \times 10^{-7} \times \exp \left(-V_{\mathrm{m}} / 7.7\right)$

$\alpha 3=3.7933 \times 10^{-7} \times \exp \left(-\mathrm{V}_{\mathrm{m}} / 7.7\right)$

$\alpha 3=3.7933 \times 10^{-7} \times \exp \left(-V_{\mathrm{m}} / 7.7\right)$

$\beta 3=\left(0.0084+0.00002 \times V_{m}\right)$

$\beta 3=\left(0.0084+0.00002 \times V_{m}\right)$

$\beta 3=\left(0.0084+0.00002 \times V_{m}\right)$

$\alpha 4=\alpha 2 / 100$

$\beta 4=\alpha 3$

$\alpha 5=\alpha 2 /\left(9.5 \times 10^{4}\right)$

$\exp (\mathrm{n})=\mathrm{e}^{\mathrm{n}}$

$\beta 5=\alpha 3 / 50$

Transition rates between modes are: background to burst $(U \rightarrow L)=0.5 \times 10^{-6} \mathrm{~ms}^{-1}$; burst to background $(\mathrm{L} \rightarrow \mathrm{U})=6.0 \times 10^{-4} \mathrm{~ms}^{-1}$.

$x$ represents $U$ or $L$ since transition rates within background or burst modes are the same.

entry into and exit from the burst mode. A diagram of single-channel records is shown (Figure 2).

The entry rate into the burst mode (burst mode entry rate) is determined by the latency to burst (time until the start of a bursting event). Using the Y1795C construct as an example, the rate constants are computed as follows: given a 500-second time course (100 milliseconds depolarization, 400 milliseconds interpulse recovery, 1,000 sweeps) of single-channel record- ing sweeps with average latency to the first burst interval $\left(\tau[\text { time constant }]_{\text {in }}=250,000\right.$ milliseconds) then the burst mode entry rate $(\mu 1)$ is computed as the reciprocal of $\tau_{\text {in }}:\left(\mu 1=4.0 \times 10^{-6} \mathrm{~ms}^{-1}\right)$. The exit rate (burst mode exit rate) is determined by the burst mode dwell time. From Figure 2, $\tau_{\text {out }}=B_{\mathrm{E}}-B_{\mathrm{S}}$, where $B_{\mathrm{E}}$ is the burst end time and $B_{\mathrm{S}}$ is the time at the start of the burst. The average $Y 1795 \mathrm{C} \tau_{\text {out }}=2.5$ seconds, and thus the burst mode exit rate $(\mu 2)$ is computed as $\mu 2=4.0 \times 10^{-4} \mathrm{~ms}^{-1}$. For WT channels, the likelihood of entry into the burst mode was very small due to infrequent bursting events, thereby making reliable extraction of entry rate constants difficult. In these cases, we estimated the rates using the relative bursting frequencies compared with Y1795C. For example, the incidence of WT bursting is approximately $20 \%$ of that observed in Y1795C, so $\mu 1$ was computed as $0.20 \times \mu 1_{\mathrm{Y} 1795 \mathrm{C}}=8.0 \times 10^{-7}$ $\mathrm{ms}^{-1}$ (Table 2). Parameter optimizations on the order of less than $10^{-7}$ for burst mode entry rates and less than $10^{-5}$ for burst mode exit rates were then implemented to exactly match the experimentally observed macroscopic amplitudes $\left(I_{\text {sus }} / I_{\text {peak }}\right)$ at the end of 100 milliseconds of depolarization (see Table 1, model parameters).

Microscopic reversibility was ensured by fixing the products of the forward and reverse transition rates in closed loops of the model. APs were computed by incorporating this model of $I_{\mathrm{Na}}$ into a previously described cellular model (15). Detailed formulation can be found in articles by Clancy and Rudy $(14,15)$.

\section{Results}

The amplitude of Y1795C mutant channel $I_{\text {sus }}$ depends on stimulation rate. Figure 3 illustrates sustained macroscopic $\mathrm{Na}^{+}$inward current $\left(I_{\text {sus }}\right)$ recorded in cells expressing Y1795C mutant $\mathrm{Na}^{+}$channels. The upper panel of the figure shows currents measured at high gain in response to the voltage protocol shown schematically in the figure. Currents recorded in response to trains of voltage pulses applied at different interpulse intervals are illustrated under steady-state conditions. The lower panel shows currents measured in this manner at two experimental interpulse intervals to mimic bradycardia (1-second interpulse interval) and tachycardia (20-millisecond interpulse interval). In all examples, a dotted line is included to indicate the zero current baseline. There is an eightfold increase in magnitude of $I_{\text {sus }}$, current that would contribute to a delay in ventricular repolarization, that occurs simply 


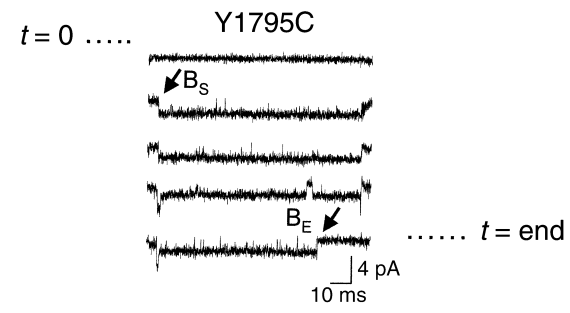

Figure 2

A diagram of single-channel records. The single-channel records allowed for derivation of theoretical rate constants of entry into and exit from the burst mode. $t$, interpulse intervals; $\mathrm{pA}$, picoAmps.

by increasing the interpulse interval. Consequently, mutation carriers would be expected to experience more severe QT prolongation at slower heart rates, consistent with clinical observations. What is the basis of this heart rate-dependent change in $I_{\text {sus }}$ at the level of single-mutant $\mathrm{Na}^{+}$channels?

The LQT-3 Y1795C mutation affects background and bursting gating modes. Figure 4 illustrates unitary current recordings from WT and Y1795C (17) mutant channels in the background gating mode during brief depolarization. Both WT and Y1795C channels open and then enter an absorbing inactivated state, as evidenced by the failure of channels to reopen. However, prolonged channel openings are apparent for Y1795C (Figure 4a, sweeps four and seven, right) compared with WT channels in the illustrated sweeps. The experimental opentime histograms (Figure 4b, top row) confirm that this is a significant effect, revealing a mutation-induced near-doubling of channel MOT (Y1795C, $0.97 \pm 0.05$ milliseconds, $n=6$; WT, $0.50 \pm 0.05$ milliseconds, $n=7$; $P<0.01)$. This time constant was extracted by fits to the experimentally determined histograms (Figure 4b, top row, dashed curves). Because transitions from open state to inactivated state (UO $\rightarrow$ UIF) dominate at this voltage $(-30 \mathrm{mV})$, the mutation-induced doubling of MOT is likely due to a reduction in the transition rate of open-state inactivation. Thus, this experimentally derived time constant was used to estimate the transition rate of open-state inactivation for mutant and WT channels in the background gating mode (Figure 4b, bottom row). The MOT is determined theoretically by the single exponential function $\mathrm{e}^{-t / \tau}$ (see Methods) where $\tau=1 /$ sum of transition rates out of the open state. These changes in open-state inactivation account for the previously reported slowing of the onset of inactivation of macroscopic Y1795C currents, but not the mutation-induced increase in $I_{\text {sus }}(17)$, since the MOT histogram indicates that all Y1795C channels inactivate within 6 milliseconds.

Figure 5 summarizes experiments in which we investigated the effects of the Y1795C mutation on singlechannel activity during prolonged depolarization to determine effects of the mutation on modal gating and to derive theoretical transition rate constants (probabilities) of entry into and exit from the burst mode. In each panel of Figure 5a, arrows are shown to indicate entry into (left arrow) and exit from (right arrow) the bursting mode during repetitively applied pulses $(-30$ $\mathrm{mV}, 100$ milliseconds, $2.0 \mathrm{~Hz}$ ). As seen in the illustrated traces in Figure 5a, bursting can be detected for WT channels, albeit infrequently (left column), but in Y1795C channels, bursting occurs more often and appears to last longer (right column). Importantly, it is apparent that once initiated, bursting can persist from pulse to pulse despite an interpulse interval (of 400 milliseconds) at $-120 \mathrm{mV}$. This finding suggests that bursting is not voltage dependent, an observation incorporated into the model.

A histogram of the latency to burst of WT and Y1795C mutant channels (Figure 5b) reflects the likelihood of entry into the burst mode. This was used to compute the burst mode entry rate $\left(\approx 10^{-6} \mathrm{~ms}^{-1}\right)$. Figure $5 c$ shows histograms of burst mode dwell times measured for WT (left) and Y1795C channels (right). Two components are apparent in both histograms. A fast component most likely reflects background reopenings, which occur infrequently for both WT and mutant channels. We estimated the dwell time in the burst mode affected by the mutation by extracting exponential fits to the slow component of the Y1795C histogram (Figure 5c). Clearly the Y1795C mutation has two effects on bursting: it increases the propensity to burst (reduced latency to burst) and the dwell time of bursting channels (increased dwell time). While both of these mutation-altered gating properties are clearly important in determining the amplitude of $I_{\text {sus }}$, are these effects at the single-channel level sufficient to account for Y1795C macroscopic channel activity? Can

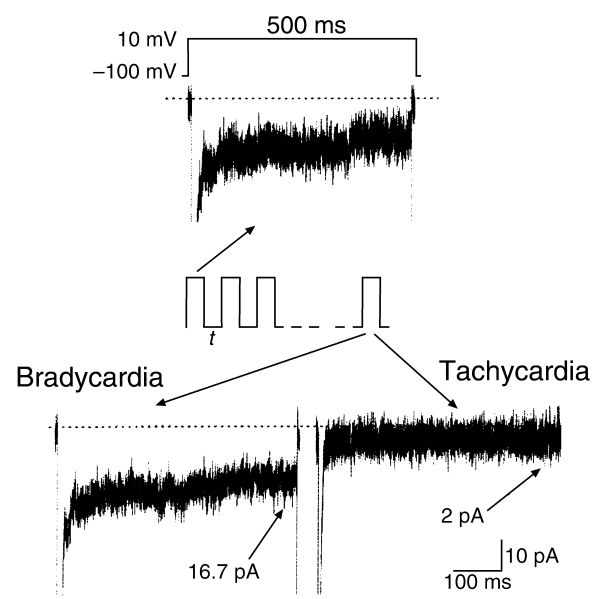

Figure 3

Influence of stimulation rate on $\mathrm{Y} 1795 \mathrm{C} I_{\text {sus. }}$. Experimental recordings of TTX-sensitive current (see Methods) are shown at high gain in cells expressing Y1795C mutant channels. Currents shown are in response to voltage pulses ( 500 milliseconds, $-10 \mathrm{mV}$ ) applied from a holding potential of $-100 \mathrm{mV}$. $\mathrm{Y} 1795 \mathrm{C} I_{\text {sus }}$ during the initial depolarizing pulse is shown in the top panel. Below are steady-state traces recorded after a train of depolarizing pulses with different interpulse intervals $(t)$ to mimic bradycardia (left, $t=1$ second) and tachycardia (right, $t=20$ milliseconds). 

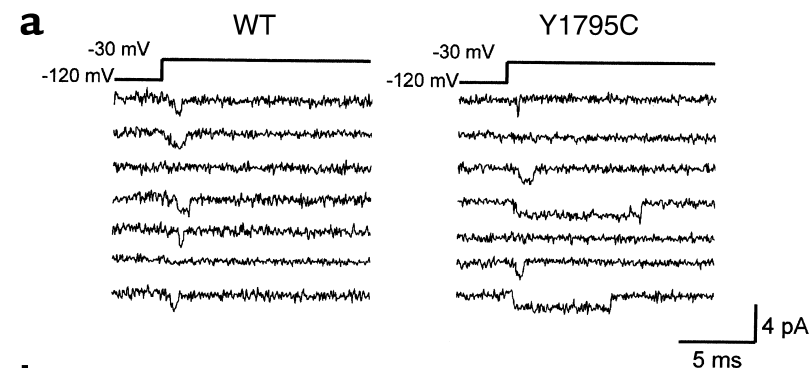

b
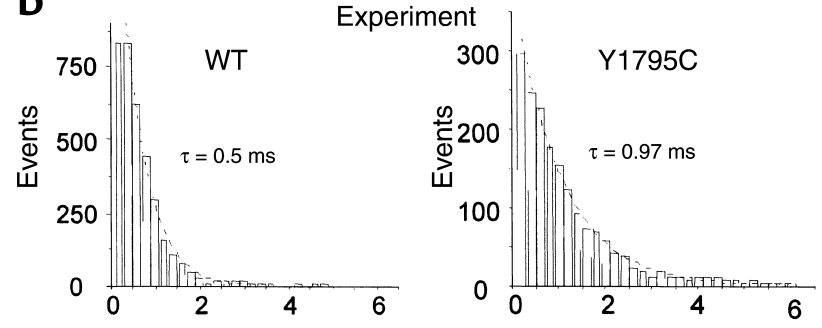

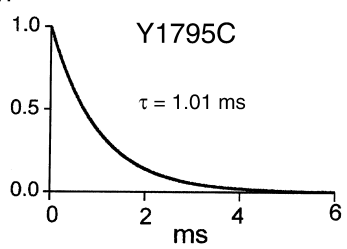

we determine whether one or both of these properties underlies the observed reverse rate dependence of $I_{\text {sus? }}$ ? Single-channel properties predict equilibrium macroscopic gating. Figure 6 compares experimental and simulated records at high gain to emphasize the combined effect of the increase in channel bursting and the reduction in the transition rate of open-state inactivation $(\mathrm{UO} \rightarrow$ UIF) on macroscopic current under steady-state conditions (100 milliseconds pulses to $-10 \mathrm{mV}, 0.5 \mathrm{~Hz}$ ). The reduced transition rate of open-state inactivation decreases the speed of decay in the simulated macroscopic Y1795C current (arrow) compared with WT cur-

\section{Figure 5}

Bursting properties of WT and $\mathrm{Y} 1795 \mathrm{C}$ mutant channels. (a) Experimental records of WT (left) and Y1795C (right) channels in a bursting mode. Arrows are included to indicate beginning (left arrow) and termination (right arrow) of channel bursting. Single-channel traces were elicited by depolarization ( $-30 \mathrm{mV}, 100$ milliseconds) from a holding potential of $-120 \mathrm{mV}$. Shown are successive sweeps for each construct. (b) Influence of the $\mathrm{Y} 1795 \mathrm{C}$ mutation on the latency to burst. The latency to burst was determined by the time to the left-hand arrows shown in a after initiation of pulses. The plots show histograms of the latency to burst (bin size $=20$ seconds, see Methods) for WT (left) and Y1795C (right) channels. A single exponential function was fitted to the histogram for $11795 \mathrm{C}$ channels, resulting in the following time constant for burst latency: $\tau_{\text {in }}=280$ seconds. A fit was not possible for WT channels (see Methods). (c) Burst mode dwell time was measured as the time between arrows in a. The plots show histograms of burst dwell times (bin size $=500$ milliseconds, see Methods) for WT (left) and Y1795C (right) channels. Double exponential functions were fit to these histograms (see text and Table 2 for values).

\section{Figure 4}

Background gating properties of WT and Y1795C mutant channels. (a) Experimentally observed WT (left) and Y1795C (right) single channels in the background mode. (b) MOTs of experimentally measured (top) and simulated (bottom) channels in the background mode (i.e., non-burst mode) at $-30 \mathrm{mV}$. Histograms (bin size $=200$ microseconds) were generated from all events (500-1,000 sweeps) for each construct. Only patches containing fewer than three channels were used to generate histograms. MOT was determined by single exponential fits to the experimental data. The time constants extracted from the fits were: $\mathrm{Y} 1795 \mathrm{C}, 0.97 \pm 0.05$ milliseconds, $n=6$; $\mathrm{WT}, 0.50 \pm 0.05$ milliseconds, $n=7$. The computation (lower panel) accurately simulates mutation-induced prolongation of MOT(Y1795C, 1.01 milliseconds; WT, 0.50 milliseconds). The MOT is determined theoretically by the single exponential function $\mathrm{e}^{-t / \tau}$ where $\tau=1 /$ sum of transition rates out of the open state. $\tau$, time constant.

rents. Notably, the decay speed in the simulation, determined from the experimental single-channel MOT, can account for the macroscopic decay observed experimentally (Figure 6, a and b, arrows, right panels). Moreover, the burst mode entry rate and burst mode exit rate derived from the single-channel records resulted in simulated sustained current amplitude very similar to that observed in the macroscopic recordings. Slight adjustments on the order of less than $10^{-7}$ for burst mode entry rate and less than $10^{-5}$ for burst mode exit rate were implemented to exactly match the experimentally observed macroscopic amplitudes $\left(I_{\text {sus }} / I_{\text {peak }}\right)$ at the end of 100 milliseconds of depolarization (see Table 1, model parameters). The Y1795C mutation
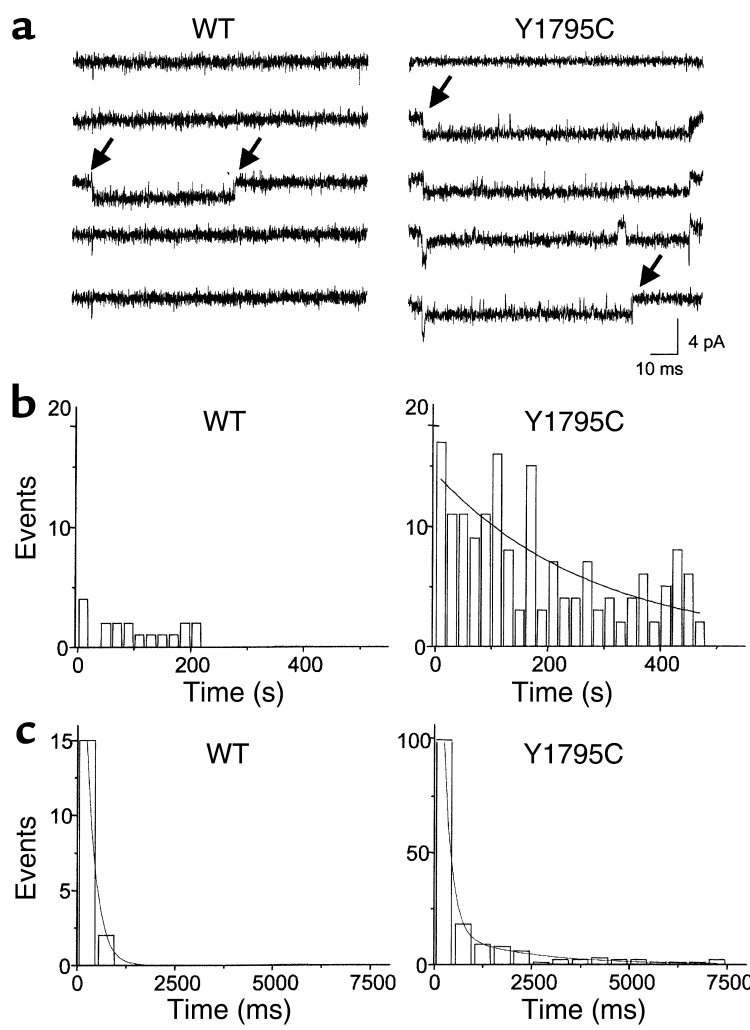
Table 2

Experimentally measured time constants and corresponding rates of entry into and exit from the burst mode

\begin{tabular}{lccc} 
& $\mathrm{WT}^{*}$ & $\mathrm{Y} 1795 \mathrm{C}$ & $\Delta \mathrm{KPQ}$ \\
$\tau_{\text {in }}(\mathrm{s})$ & $\mathrm{NA}$ & 250 & 180 \\
Rate $_{\text {in }}\left(\mathrm{ms}^{-1}\right)$ & $8.0 \times 10^{-7}$ & $4.0 \times 10^{-6}$ & $5.6 \times 10^{-6}$ \\
$\tau_{\text {out }}(\mathrm{s})$ & 0.2 & 2.5 & 3.6 \\
Rate $_{\text {out }}\left(\mathrm{ms}^{-1}\right)$ & $5 \times 10^{-3}$ & $4.0 \times 10^{-4}$ & $2.8 \times 10^{-4}$ \\
\hline
\end{tabular}

*WT rates are estimated as described in Methods.

results in a simulated increase of approximately fivefold in sustained current compared with WT ( $0.5 \%$ vs. $0.1 \%$, respectively), in agreement with experimentally determined currents (shown in Figure 6c).

These changes in MOT and burst mode gating do not affect computed voltage dependence of activation or inactivation (Figure 7 , a and b, right panels), consistent with experimental measurements for Y1795C channels (Figure 7, a and b, left panels). However, the increase in experimentally determined single-channel MOT, which is due to reduced transition rates of open-state inactivation, is simulated by the model to result in an increase in macroscopic current density at membrane potentials above the inactivation threshold (above $-40 \mathrm{mV}$ ) (Figure $7 \mathrm{c}$, right). The longer MOTs of the mutant channels afford greater opportunity for the passage of $\mathrm{Na}^{+}$, thereby resulting in larger current density. The $15 \%$ increase simulated by the model fits the experimentally observed trend (a 20\% increase) shown in Figure 7c, left. Thus the model, with critical parameters determined solely from MOT and burst mode gating experiments, accurately simulates steady-state macroscopic current properties. We next tested model predictions of channel activity under nonequilibrium conditions.

Single-channel gating mode kinetics account for stimulation rate-dependent changes in $I_{\text {sus }}$. We tested the predictions of our model based on experimentally obtained kinetic

\footnotetext{
Figure 6

Experimental and simulated macroscopic sustained current. (a) Shown are experimental recordings of TTX-sensitive currents in response to depolarization (-10 $\mathrm{mV}, 100$ milliseconds) from a -100 $\mathrm{mV}$ holding potential recorded in cells expressing WT (left) and Y1795C (right) channels. (b) Simulated WT (left) and Y1795C (right) currents generated using the $\mathrm{Na}^{+}$channel Markov model shown in Figure 1. The reduced rate of open channel inactivation (UO $\rightarrow$ UIF) due to $\mathrm{Y} 1795 \mathrm{C}$ is reflected in the longer time course of the macroscopic current decay ( $\mathbf{a}$ and $\mathbf{b}$, arrows). (c) Sustained current, normalized to peak current, is plotted as percent peak current for WT and $\mathrm{Y} 1795 \mathrm{C}$ channels. The left panel is a summary of experimentally recorded $I_{\text {sus }}(-10 \mathrm{mV}, 100$ milliseconds). The values are: $\mathrm{WT}$, $0.07 \% \pm 0.01 \%, n=7 ; \mathrm{Y} 1795 \mathrm{C}, 0.40 \% \pm 0.04 \%, n=10$. The right panel shows the values simulated by the model using the gating parameters described in the text. In both the experimentally recorded and simulated currents, the sustained current $\left(I_{\text {sus }}\right)$ relative to peak current $\left(I_{\text {peak }}\right)$ is increased in $\mathrm{Y} 1795 \mathrm{C}\left(I_{\text {sus } / \text { peak }}=0.5 \%\right)$ compared with $\operatorname{WT}\left(I_{\text {sus }} / I_{\text {peak }} \leq 0.1 \%\right)$.
}

parameters under pacing conditions (i.e., nonequilibrium gating). Figure 8 compares experimental (left panels) and simulated (right panels) macroscopic Y1795C mutant current shown during trains of 20 pulses $(-10$ $\mathrm{mV}$ for 500 milliseconds with interpulse voltage of $-100 \mathrm{mV}$ ). Currents measured in response to pulses applied at an interpulse interval of 20 milliseconds are shown in Figure 8, a and b. Each graph shows the superposition of current in response to the first and 20 th pulses in the train. After 20 pulses (Figure 8a), $I_{\text {sus }}$ is markedly reduced in both the experimental (left) and theoretical (right) records. The experimentally measured percentage of $I_{\text {sus }}$ at the end of each 500 -millisecond depolarization $\left(I_{\text {susn }}\right)$ relative to the $I_{\text {sus }}$ amplitude of the first pulse $\left(I_{\text {sus } 1}\right)$ is summarized in Figure $8 \mathrm{~b}$, left, as $\left(I_{\text {susn }} / I_{\text {sus } 1}\right) \times 100$. The corresponding simulation is shown in Figure 8b, right, indicating good agreement between theory and experiment. This pulse-dependent reduction in $I_{\text {sus }}$ is less in both experimental and simulated records when the interpulse interval is increased to 1 second. These results confirm that $I_{\text {sus }}$ carried by Y1795C channels is augmented at slow heart rates, and furthermore, that the single-channel data incorporated into the model are sufficient to account for the stimulation-rate dependence of $I_{\text {sus. }}$.

Are the burst mode entry rate and the burst mode exit rate equally important in determining the stimulation-rate dependence of $I_{\text {sus }}$ ? In order to determine whether the burst mode entry rate (latency to burst) or the burst mode exit rate (burst mode dwell time) determines rate-dependent changes in $I_{\text {sus }}$, we simulated a range of possible burst mode entry and exit rates. If one transition rate is exclusively responsible for the stimulation rate-dependent changes in $I_{\text {sus }}$, then variation in the other transition rate should not affect stimulation-rate dependence of $I_{\text {sus }}$.

Using the model, we tested this idea (Figure 9) at two stimulation rates with the fast (20-millisecond inter-
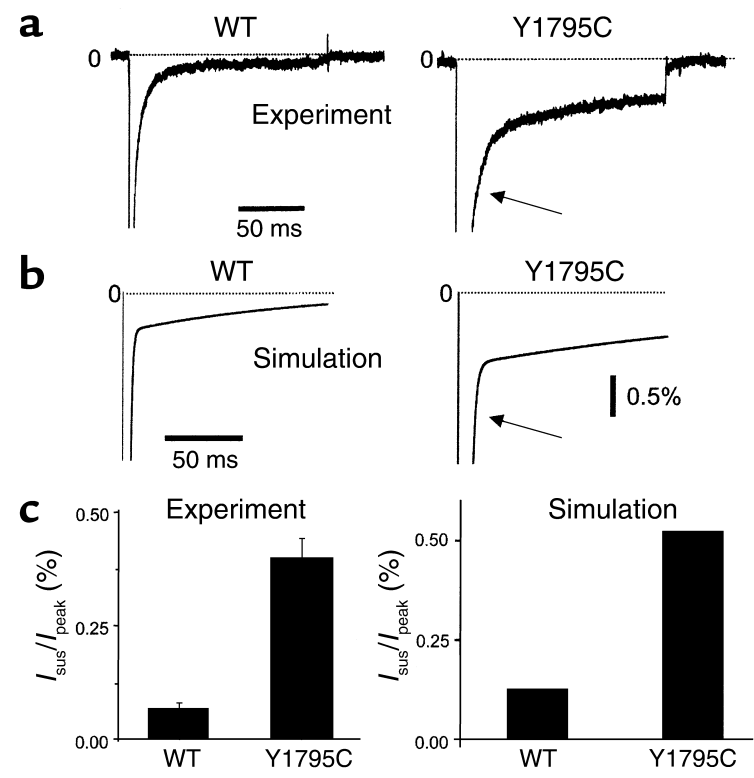

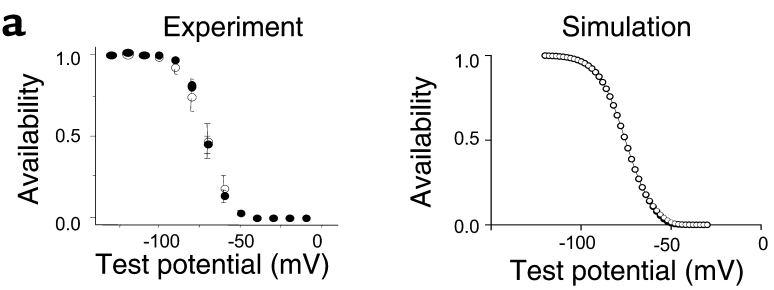

b
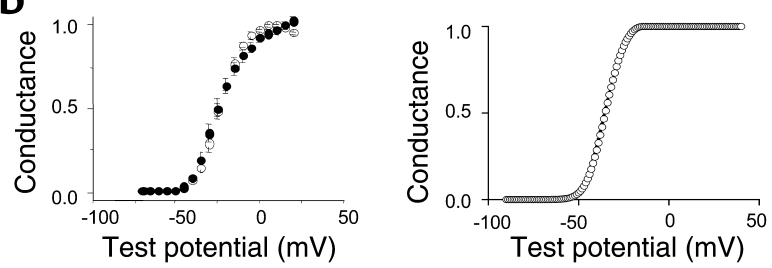

C

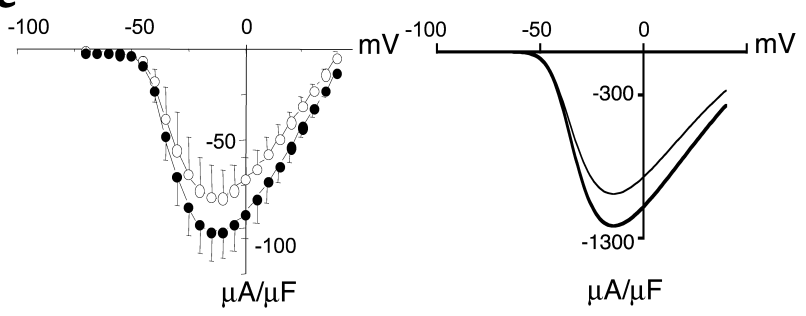

pulse interval, left) and slow (1-second interpulse interval, right) protocols described for Figure 8 . The burst mode entry rate was fixed at $10^{-4}, 10^{-5}$, or $10^{-6} \mathrm{~ms}^{-1}(10)$, while each graph shows three burst mode exit rates $\left(10^{-6} \mathrm{~ms}^{-1}\right.$ to $\left.10^{-4} \mathrm{~ms}^{-1}\right)$. At the fast stimulation rate (left), $I_{\text {sus }}$ does not decrease in a pulse-dependent manner when the burst mode exit rate is $10^{-6} \mathrm{~ms}^{-1}$, regardless of the burst mode entry rate (Figure 9, a-c, open circles). In contrast, increasing the burst mode exit rate $\left(10^{-5} \mathrm{~ms}^{-1}\right.$ and $\left.10^{-4} \mathrm{~ms}^{-1}\right)$ results in pulse-dependent reduction of $I_{\text {sus }}$ at each of the three entry rates (Figure 9 , a-c, left panels). These simulations suggest that the burst mode exit rate primarily determines the rate dependence of $I_{\text {sus. }}$.

In Figure 9, a-c, right panels, the simulations were repeated at the slow stimulation rate as described above. While the stimulation rate-dependent reduction in $I_{\text {sus }}$

\section{Figure 8}

Stimulation-rate dependence of Y1795C channel sustained current $\left(I_{\text {sus }}\right)$ : agreement between simulated and experimental data. (a) The panels compare experimental (left) and simulated (right) records of Y1795C channel sustained current $\left(I_{\text {sus }}\right)$ during trains (20 pulses) of repetitive voltage pulses applied after a pulse-free period. Shown is the superposition of current in response to the first and 20th pulses $(-10$ $\mathrm{mV}, 500$ milliseconds). The experimental records are TTX-sensitive currents (see Methods). The interpulse interval was 20 milliseconds. (b) Pulse-dependent changes in $I_{\text {sus }}$ during rapid stimulation. $I_{\text {sus }}$ during successive pulses $\left(I_{\text {susn }}\right)$ was normalized to $I_{\text {sus }}$ recorded (left) or computed (right) during the first pulse $\left(I_{\text {sus } 1}\right)$ of the pulse train and plotted versus pulse number. The interpulse interval was 20 milliseconds. (c) Pulse-dependent changes in $I_{\text {sus }}$ in response to slow stimulation rate. $I_{\text {sus }}$ was measured, computed, and plotted as in $\mathbf{b}$, but in this case the interpulse interval was 1 second. CL, cycle length.

\section{Figure 7}

Macroscopic current properties of experimental and simulated WT and Y1795C channels. Shown in each panel is a summary of experimentally determined records (left) and simulated current (right). In the experimental records, open circles are WT $(n=8)$ and filled circles are Y1795C $(n=9)$ channels. Theoretical symbols overlap except in $\mathbf{c}$ (where the thick line represents Y1795C channels in the right panel). (a) Shown is the voltage dependence of inactivation following 500-millisecond conditioning pulses. Normalized current is plotted versus conditioning pulse voltage. (b) The Y1795C mutation does not affect the voltage dependence of activation (see Methods). (c) The Y1795C mutation increases peak experimentally determined (left) and computed current (right). Experimental currents were measured in lowered extracellular $\mathrm{Na}^{+}$to ensure voltage control. In the simulation, the slowing of the transition rate from open to inactivation states acts to increase open-state probability in the Y1795C mutant compared with $\mathrm{WT}$, resulting in an increase in macroscopic $I_{\mathrm{Na}}$. WT macroscopic $I_{\mathrm{Na}}$ is about $20 \%$ and $15 \%$ less than $\mathrm{Y} 1795 \mathrm{C} I_{\mathrm{Na}}$ in the experiment and the simulation, respectively. Note that simulated density is larger because it is computed in a simulated cardiac myocyte in full $\mathrm{Na}^{+}$solutions. $\mu \mathrm{A}$, microAmps; $\mu \mathrm{F}$, microfarad.

is much weaker than at the fast rate, it is again primarily determined by the burst mode exit rate. The simulations strongly suggest that it is the burst mode exit rate calculated from the burst mode dwell time, rather than burst mode entry rate determined by the latency to burst, that mainly governs the rate dependence of $I_{\text {sus. }}$.

To validate this prediction, we investigated the stimulation-rate dependence of $I_{\text {sus }}$ and single-channel bursting in another LQT-3 mutation, $\triangle \mathrm{KPQ}$, the first LQT-3 mutation reported to promote channel bursting $(5,7)$. Figure 10 illustrates experimental data for the $\triangle \mathrm{KPQ}$ channels, and Table 2 summarizes key findings for both constructs studied. The records for the $\triangle \mathrm{KPQ}$ channels shown in Figure 10 strongly resemble those for the Y1795C channel: channel bursting is

\section{a}

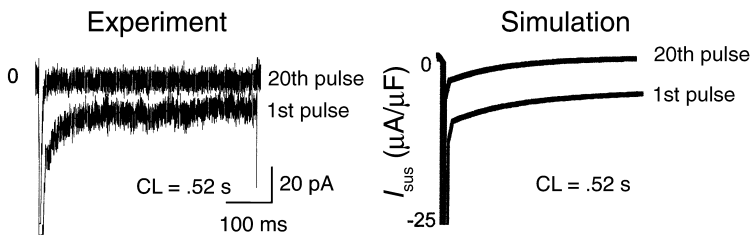

b $\mathrm{CL}=.52 \mathrm{~s}$
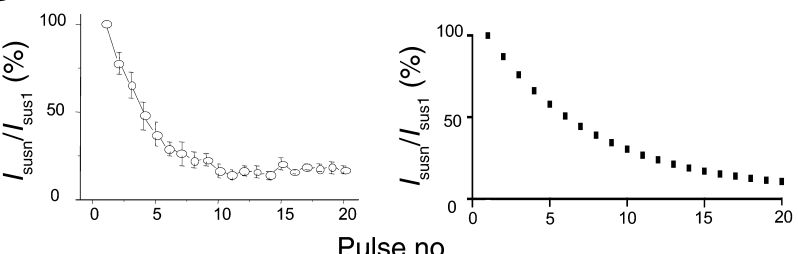

Pulse no.

C $\mathrm{CL}=1.5 \mathrm{~s}$

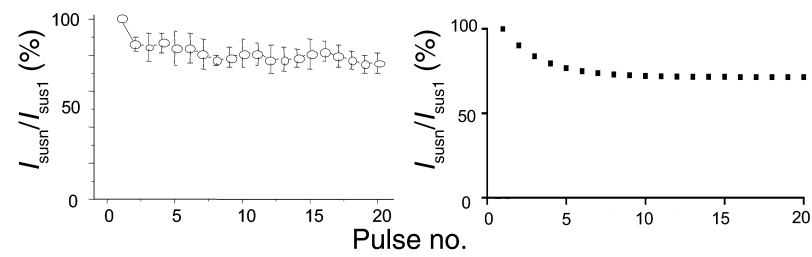


Fast rate $=0.52 \mathrm{~s}$
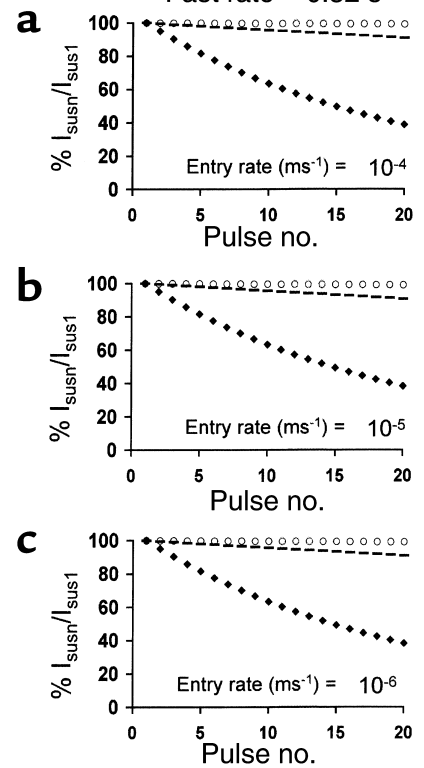

Burst mode exit rate $\left(\mathrm{ms}^{-1}\right)=$ $10^{-4}$
Slow rate $=1.5 \mathrm{~s}$
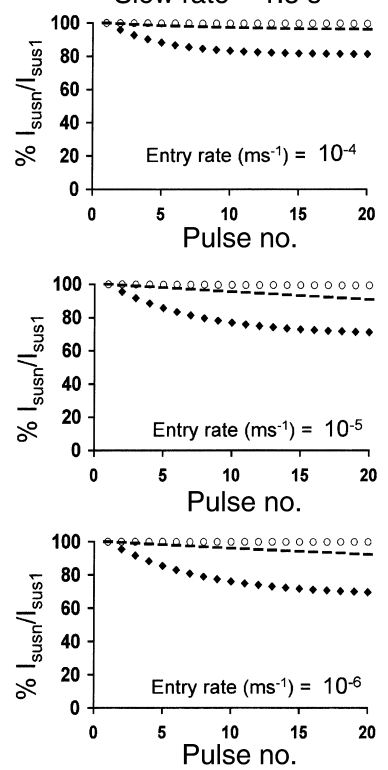

$10^{-5}-10^{-6} \mathrm{O}$

apparent (Figure 10a) and the mutation increases the dwell time in the burst mode (Figure 10b). $\triangle \mathrm{KPQ}$ channels burst more readily than do Y1795C channels, as indicated by the reduced latency to burst for $\triangle \mathrm{KPQ}$ channels (Table 2). Macroscopic $\triangle \mathrm{KPQ}$ currents measured in response to repetitive pulsing decrease in a pulse-by-pulse manner remarkably similar to Y1795C channels (Figure 10, c and d). Like the Y1795C mutation, the $\triangle \mathrm{KPQ}$ mutation reduces the burst mode exit rate (increases the burst mode dwell time, Table 2), and consistent with predictions of the model, exhibits a very similar rate-dependent reduction in $I_{\text {sus }}$ (Figure $10 \mathrm{~d})$. Thus, these results support the theoretical analysis and indicate that mutation-induced change in the burst mode exit rate is the primary determinant of stimulation-rate dependence of $I_{\text {sus. }}$.

Mutation-induced changes in single-channel kinetics account for inverse rate-dependent prolongation of APD. Finally, we investigated the effect of the Y1795C

\section{Figure 10}

Experimental investigation of the reversed stimulation dependence of $\triangle \mathrm{KPQ} I_{\text {sus. }}$ (a) Cell-attached single-channel activity in a cell expressing $\triangle K P Q$ channels. Shown are consecutive sweeps. (b) The dwell time of bursting activity was measured as in Figure 5 and was used to generate the histogram shown (bin size $=500$ milliseconds). The smooth curve is the best fit double exponential function best fit to the histogram (see Table 2 for values). (c) TTX-sensitive macroscopic current is shown at high gain in response to the first and 20th pulses $(-10 \mathrm{mV}, 500$ milliseconds, holding potential $-100 \mathrm{mV})$ of a pulse train (20-millisecond interpulse interval). (d) Mean $\pm \mathrm{SEM} \triangle \mathrm{KPQ} I_{\text {sus }}$ (open circles, $n=6$ ) normalized to $I_{\text {sus } 1}$ and plotted as a function of pulse number during the pulse train (interpulse interval, 20 milliseconds). The dotted line replots the experimental data for Y1795C channels shown in Figure $8 \mathrm{~b}$ for comparison.

\section{Figure 9}

Simulations predict that the burst mode dwell time, rather than latency to burst, determines the stimulation-rate dependence of $I_{\text {sus }}$. The figure illustrates the effect on $I_{\text {sus }}$ of changing the burst mode exit rates (open circles, $10^{-6} \mathrm{~ms}^{-1}$; dashes, $10^{-5} \mathrm{~ms}^{-1}$; filled diamonds, $10^{-4} \mathrm{~ms}^{-1}$ ) with fixed entry rates. In each panel, computations are shown for fast (interpulse interval, 20 milliseconds, left) and slow (interpulse interval, 1 second, right) stimulation rates (protocol described in Figure 8 legend). $I_{\text {sus }}$ at the end of each depolarization pulse $\left(I_{\text {susn }}\right)$ is plotted as a percentage of $I_{\text {sus }}$ at the end of the first pulse $\left(I_{\text {sus } 1}\right)$. The pulse-dependent reduction of $I_{\text {sus }}$ for each burst mode exit rate is unchanged regardless of the fixed entry rate $\left(10^{-4} \mathrm{~ms}^{-1}\right.$ in $\mathbf{a}, 10^{-5} \mathrm{~ms}^{-1}$ in $\mathbf{b}$, and $10^{-6} \mathrm{~ms}^{-1}$ in $\left.\mathbf{c}\right)$.

mutation on the rate dependence of APD using a theoretical model of the cardiac ventricular AP during pacing (15). Figure 11 illustrates the 19th and 20th APs (upper rows) and corresponding $I_{\mathrm{Na}}$ high gain (lower rows) from virtual cells containing WT (left) and Y1795C (right) channels. Figure 11a compares APs and currents after pacing at a cycle length of 300 milliseconds from resting steady state (see Methods). The Y1795C mutation clearly results in larger $I_{\text {sus }}$ (arrow) compared with WT that becomes progressively larger as the pacing rate is slowed (Figure 11b, cycle length of 1,200 milliseconds). This stimulation rate-dependent increase of $I_{\text {sus }}$ in the mutant channel underlies the preferential APD prolongation at slow rates, a result that is consistent with the clinical observation that Y1795C carriers are susceptible to arrhythmia events during bradycardia.

\section{Discussion}

Mutation-induced alteration in modal gating dwell time underlies inverse rate-dependent increase in macroscopic current. Rhythm disturbances resulting from gene-specific mutation-induced long QT syndrome vary widely in response to heart rate $(1,16)$. LQT-1 mutations due to
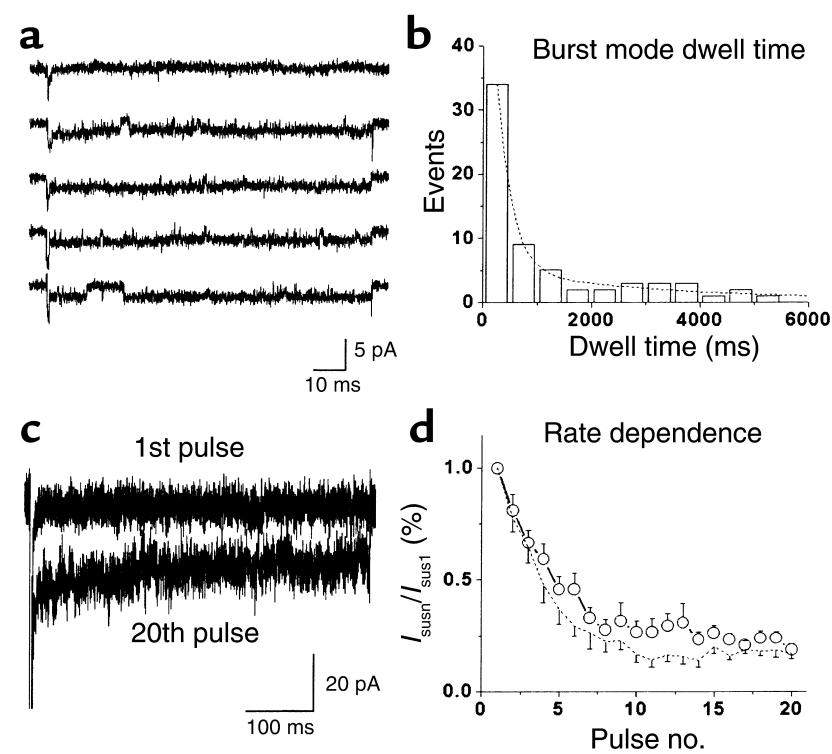
a

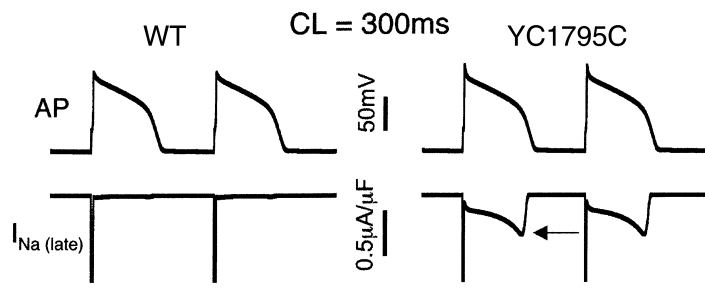

b

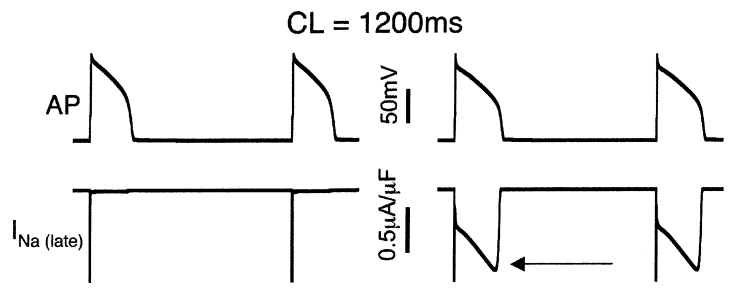

C

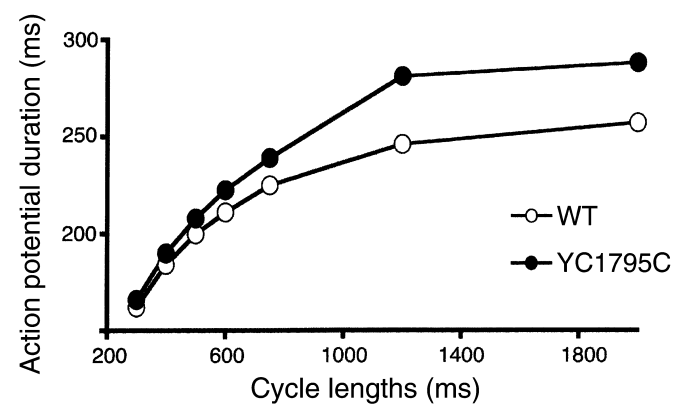

defects in KvLQT1 are strongly associated with $\beta$-adrenergic stimulation and cardiac events during rapid pacing $(1,22)$. Recently, it has been demonstrated that a frequently occurring LQT-1 mutation results in the disruption of a signaling complex required for channel phosphorylation that increases the amplitude (23) of the slowly activating component of the delayed rectifier $\mathrm{K}^{+}$current $\left(I_{\mathrm{Ks}}\right)$. The signaling complex is crucial for APD shortening during rapid pacing. LQT-2 patients that have defects in HERG are equally likely to exhibit cardiac events at fast and slow rates (1) that likely stem from different mechanisms (24). $\mathrm{Na}^{+}$channel-linked LQT-3 symptoms typically manifest at slower heart rates where patient phenotypes are marked by rhythm disturbances that may act as precursors to sudden cardiac death (1), although a recent study in the $\triangle \mathrm{KPQ}$ mutation expressed in a transgenic mouse model demonstrated arrhythmogenic events at very fast pacing rates (25). The understanding of the mechanism of these variable rate dependencies is crucial for the development of therapeutic interventions.

Previous analysis of an LQT-3 mutant $\mathrm{Na}^{+}$channel (Y1795C) demonstrated that the persistent non-inactivating $I_{\mathrm{Na}}, I_{\mathrm{sus}}$, exhibits implicit stimulation-rate dependence even in the absence of neural influence (17). These results suggest that gating properties of $I_{\mathrm{Na}}$ may be sufficient to account for the stimulation-rate dependence of $I_{\text {sus. }}$. In this study we have combined experimental and theoretical approaches in order to elucidate the mechanism of the stimulation rate-depend-

\section{Figure 11}

The reverse stimulation-rate dependence of $Y 1795 \mathrm{C}$ on $I_{\text {sus }}$ and APD. (a) The 19th and 20th APs from virtual cells containing WT (left) and $\mathrm{Y} 1795 \mathrm{C}$ (right) channels with corresponding $/ \mathrm{Na}$ (at high gain, bottom) (see Methods). The Y1795C mutation clearly results in larger $I_{\text {sus }}$ (arrow) compared with WT that becomes progressively larger as the stimulation rate is slowed, as indicated by the APs shown in (b) (stimulation rate, 1,200 milliseconds). (c) Computed APD for cells expressing WT and $\mathrm{Y} 1795 \mathrm{C}$ channels plotted against simulated stimulation cycle length $(C L)$. As the cycle length becomes progressively longer (simulating slowing of heart rate), the WT and Y1795C adaptation curves diverge, indicating that the $\mathrm{Y} 1795 \mathrm{C}$ mutation exerts its deleterious effects preferentially at slow stimulation rates.

ent reduction in $I_{\text {sus }}$ associated with the bradycardia dependence of $\mathrm{Na}^{+}$channel-linked long QT syndrome. Recordings from single channels allow for the derivation of theoretical entry rates into and exit rates from the bursting mode of gating. Our results indicate that mutation-induced changes in the amount of time that mutant channels spend bursting (burst mode dwell time), rather than how readily they burst (latency to burst), primarily determine the inverse heart-rate dependence of $I_{\text {sus. }}$. We experimentally confirmed this prediction by analysis of $\triangle \mathrm{KPQ}$ mutant channels for which the burst mode exit rate (determined by the burst mode dwell time) was found to be very similar to the derived rate for Y1795C channels. Based on our theoretical analysis, this suggested that the stimulation rate-dependent reduction in $I_{\text {sus }}$ should be similar for $\triangle \mathrm{KPQ}$ and Y1795C channels, a prediction confirmed by the data presented in Figure 10d. Thus pharmacological control of the burst mode exit rate becomes a novel therapeutic strategy for rate-dependent regulation of APD in the heart (see below for structural targets).

Mechanistic insights from the computational model. The cardiac ventricular AP is a complex cellular event with multiple interconnected pathways contributing to the balance of ionic currents that control its waveform (26). Hence, extrapolation from mutation-induced changes in gating of a single ion channel to effects on AP waveform is not obvious, and in many cases requires computer-based simulations that can estimate the contributions from multiple pathways (27). It must be noted, however, that modeling of complex biological processes is not without limitations. By definition, a model is a simplification of the actual biological process that allows insight and understanding but may result in the exclusion of details necessary for absolute understanding of biological complexity and mechanism $(28,29)$. Nonetheless, the value of this approach is illustrated by the results presented in Figure 11. We demonstrated that the Y1795C channels result in larger macroscopic $I_{\text {sus }}$ than do WT channels, and that this current becomes progressively larger as the stimulation rate is slowed. However, at sufficiently rapid stimulation rates (Figure 11a), despite the larger mutant-channel sustained inward current (Figure 11a, arrow), the mutation has little effect on APD 
(compare WT and Y1795C APs in Figure 11a). What mechanism underlies this apparent contradiction?

The simulation suggests that it is the summation of the mutation-induced inward current along with critical time-dependent potassium current that yields the overall rate-dependent effects of the mutation on the AP. As the stimulation rates become progressively slower, the WT and Y1795C adaptation curves diverge (Figure 11c), indicating that the Y1795C mutation exerts its deleterious effects preferentially at slow rates. At fast rates, $I_{\mathrm{Ks}}$ dominates the rate-dependent adaptation of APD $(30,31)$. Fast pacing results in short diastolic intervals that prevent complete deactivation of $I_{\mathrm{Ks}}$, resulting in the buildup of instantaneous $I_{\mathrm{Ks}}$ repolarizing current at the AP onset. This effect overwhelms $I_{\text {sus }}$ caused by Y1795C. At slower rates, less repolarizing current exists during each AP due to sufficient time between beats to allow complete deactivation of $I_{\mathrm{Ks}}$. This effect, coupled with the reverse rate-dependent increases in $I_{\text {sus }}$ due to Y1795C, gives rise to preferential prolongation at slow rates, consistent with the clinical observation that $\mathrm{Y} 1795 \mathrm{C}$ carriers are susceptible to arrhythmia events during bradycardia.

Insight into structural control of channel bursting: novel targets for drug development. The structural basis of failed inactivation is poorly understood. Mutations in the III-IV linker region, both naturally occurring and experimentally induced, have been shown to disrupt the inactivation process (32) and underlie long QT syndrome $(7,12)$. The III-IV linker is thought to act as a blocking particle that moves into and occludes the pore following voltage-induced channel opening. More recently, LQT mutations in the C-terminus of SCN5A have been shown to affect inactivation gating, thereby implicating a role for the C-terminus in channel inactivation $(17,18,33,34)$. This focus on a possible role of the C-terminus in control of inactivation has been extended by chimeric analysis of brain and heart $\mathrm{Na}^{+}$channels (35), site-directed mutagenesis of cardiac $\mathrm{Na}^{+}$channels (36), and a structural analysis of the C-terminal tail of the cardiac $\mathrm{Na}^{+}$channel (37).

Analysis of mutations $\mathrm{Y} 1795 \mathrm{C}$ and $\Delta \mathrm{KPQ}$ provides insight into a possible structural basis for mutationinduced modulation of inactivation that is reflected in modal gating. It is possible that regions of the C-terminus act as a "docking station" for the III-IV linker at voltages that are unfavorable to inactivation. During depolarization, allosteric movements may help to release the linker and allow blockage of the pore. In this way, a charged portion of the C-terminus may act as an inactivation voltage sensor. Allosteric movement of the sensor, induced by depolarization, might release the blocking particle, allowing for inactivation. Mutations in this region may disrupt the sensor, making release of the linker less likely, thereby increasing the likelihood of failed inactivation. This is consistent with the proposal that the inactivation particle is semipermanently docked when channels are closed and that this docking interaction may be modified by some C-terminal mutations. The nearly identical effects of the $\triangle \mathrm{KPQ}$ and Y1795C mutations on burst mode dwell time provide evidence for the symmetry in C-terminus and III-IV linker interactions in the control of inactivation gating.

Understanding the structural basis of the mechanism of $\mathrm{Na}^{+}$channel gating properties is crucial for the development of specifically targeted pharmacological interventions, and the present results strongly suggest that either the $\mathrm{C}$-terminus and/or the inactivation gate (III-IV linker) are candidate molecular targets for the development of drugs intended to control APD in the heart, not by blocking of potassium channels, but through modulation of $\mathrm{Na}^{+}$channels via control of modal gating.

\section{Acknowledgments}

This work was supported by NIH National Heart Lung and Blood Institute grants R01 HL-56810-05 and P01 HL-67849-01.

1. Schwartz, P.J., et al. 2001. Genotype-phenotype correlation in the longQT syndrome: gene-specific triggers for life-threatening arrhythmias. Circulation. 103:89-95.

2. Roden, D.M., and Spooner, P.M. 1999. Inherited long QT syndromes: a paradigm for understanding arrhythmogenesis. J. Cardiovasc. Electrophysiol. 10:1664-1683.

3. Choi, J.Y., et al. 2001. Aberrant CFTR-dependent HCO3- transport in mutations associated with cystic fibrosis. Nature. 410:94-97.

4. Chen, Q., et al. 1998. Genetic basis and molecular mechanism for idiopathic ventricular fibrillation. Nature. 392:293-296.

5. Wang, Q., et al. 1995. SCN5A mutations associated with an inherited cardiac arrhythmia, long QT syndrome. Cell. 80:805-811.

6. Sanguinetti, M.C., Jiang, C., Curran, M.E., and Keating, M.T. 1995. A mechanistic link between an inherited and an acquired cardiac arrhythmia: HERG encodes the $\mathrm{I}_{\mathrm{Kr}}$ potassium channel. Cell. 81:299-307.

7. Bennett, P.B., Yazawa, K., Makita, N., and George, A.L. 1995. Molecular mechanism for an inherited cardiac arrhythmia. Nature. 376:683-685.

8. Shalaby, F.Y., et al. 1997. Dominant-negative KvLQT1 mutations underlie the LQT1 form of long QT syndrome. Circulation. 96:1733-1736.

9. Splawski, I., et al. 2000. Spectrum of mutations in long-QT syndrome genes: KVLQT1, HERG, SCN5A, KCNE1, and KCNE2. Circulation. 102:1178-1185.

10. Moss, A.J., et al. 1991. The long QT syndrome: prospective longitudinal study of 328 families. Circulation. 84:1136-1144.

11. Keating, M.T., and Sanguinetti, M.C. 2001. Molecular and cellular mechanisms of cardiac arrhythmias. Cell. 104:569-580.

12. Chandra, R., Starmer, C.F., and Grant, A.O. 1998. Multiple effects of KPQ deletion mutation on gating of human cardiac Na+ channels expressed in mammalian cells. Am. J. Physiol. 274:H1643-H1654.

13. Dumaine, R., et al. 1996. Multiple mechanisms of $\mathrm{Na}+$ channel linked long-QT syndrome. Circ. Res. 78:916-924.

14. Clancy, C.E., and Rudy, Y. 1999. Linking a genetic defect to its cellular phenotype in a cardiac arrhythmia. Nature. 400:566-569.

15. Clancy, C.E., and Rudy, Y. 2002. $\mathrm{Na}(+)$ channel mutation that causes both Brugada and long-QT syndrome phenotypes: a simulation study of mechanism. Circulation. 105:1208-1213.

16. Schwartz, P.J., et al. 1995. Long QT syndrome patients with mutations of the SCN5A and HERG genes have differential responses to NA+ channel blockade and to increases in heart rate: implications for genespecific therapy. Circulation. 92:3381-3386.

17. Rivolta, I., et al. 2001. Inherited Brugada and LQT-3 syndrome mutations of a single residue of the cardiac sodium channel confer distinct channel and clinical phenotypes. J. Biol. Chem. 276:30623-30630.

18. An, R.H., et al. 1998. Novel LQT-3 mutation affects $\mathrm{Na}+$ channel activity through interactions between alpha- and beta1-subunits. Circ. Res. 83:141-146.

19. Premkumar, L.S., Qin, F., and Auerbach, A. 1997. Subconductance states of a mutant NMDA receptor channel kinetics, calcium, and voltage dependence. J. Gen. Physiol. 109:181-189.

20. Qin, F., Auerbach, A., and Sachs, F. 1996. Estimating single-channel kinetic parameters from idealized patch-clamp data containing missed events. Biophys. J. 70:264-280. 
21. Berman, M.F., Camardo, J.S., Robinson, R.B., and Siegelbaum, S.A. 1989. Single sodium channels from canine ventricular myocytes: voltage dependence and relative rates of activation and inactivation. J. Physiol. 415:503-531.

22. Moss, A.J., et al. 2000. Effectiveness and limitations of beta-blocker therapy in congenital long-QT syndrome. Circulation. 101:616-623.

23. Marx, S.O., et al. 2002. Requirement of a macromolecular signaling complex for beta adrenergic receptor modulation of the KCNQ1KCNE1 potassium channel. Science. 295:496-499.

24. Clancy, C.E., and Rudy, Y. 2001. Cellular consequences of HERG mutations in the long QT syndrome: precursors to sudden cardiac death. Cardiovasc. Res. 50:301-313.

25. Nuyens, D., et al. 2001. Abrupt rate accelerations or premature beats cause life-threatening arrhythmias in mice with long-QT3 syndrome. Nat. Med. 7:1021-1027.

26. Rivolta, I., Abriel, H., and Kass, R.S. 2001. Ion channels as targets for drugs. In Cell physiology sourcebook. N. Sperelakis, editor. Academic Press. New York, New York, USA. 643-652.

27. Luo, C.H., and Rudy, Y. 1994. A dynamic model of the cardiac ventricular action potential. I. Simulations of ionic currents and concentration changes. Circ. Res. 74:1071-1096.

28. Noble, D. 2002. Modeling the heart - from genes to cells to the whole organ. Science. 295:1678-1682.

29. Noble, D. 2002. The rise of computational biology. Nat. Rev. Mol. Cell Biol. 3:459-463.
30. Romey, G., et al. 1997. Molecular mechanism and functional significance of the MinK control of the KvLQT1 channel activity. J. Biol. Chem. 272:16713-16716.

31. Faber, G.M., and Rudy, Y. 2000. Action potential and contractility changes in $[\mathrm{Na}(+)](\mathrm{i})$ overloaded cardiac myocytes: a simulation study. Biophys. J. 78:2392-2404.

32. Kimbrough, J.T., and Gingrich, K.J. 2000. Quaternary ammonium block of mutant $\mathrm{Na}+$ channels lacking inactivation: features of a transition-intermediate mechanism. J. Physiol. 529:93-106.

33. Bezzina, C., et al. 1999. A single $\mathrm{Na}(+)$ channel mutation causing both long-QT and Brugada syndromes. Circ. Res. 85:1206-1213.

34. Wehrens, X.H., Abriel, H., Cabo, C., Benhorin, J., and Kass, R.S. 2000 Arrhythmogenic mechanism of an LQT-3 mutation of the human heart $\mathrm{Na}(+)$ channel alpha-subunit: a computational analysis. Circulation. 102:584-590.

35. Mantegazza, M., Yu, F.H., Catterall, W.A., and Scheuer, T. 2001. Role of the C-terminal domain in inactivation of brain and cardiac sodium channels. Proc. Natl. Acad. Sci. USA. 98:15348-15353.

36. Deschenes, I., Trottier, E., and Chahine, M. 2001. Implication of the C-terminal region of the alpha-subunit of voltage-gated sodium channels in fast inactivation. J. Membr. Biol. 183:103-114.

37. Cormier, J.W., Rivolta, I., Tateyama, M., Yang, A.S., and Kass, R.S. 2002 Secondary structure of the human cardiac $\mathrm{Na}+$ channel $\mathrm{C}$ terminus. Evidence for a role of helical structures in modulation of channel inactivation. J. Biol. Chem. 277:9233-9241. 